\title{
Link between FGF21 and blood pressure
}

Circulating levels of fibroblast growth factor 21 (FGF21) are

known to be associated with hypertension in humans, and administration of FGF21 ameliorates high blood pressure in a rat model of hypertension. However, the mechanisms underlying this association are unclear. Now, new research suggests that FGF21 activates the angiotensin-converting enzyme 2 (ACE2)-angiotensin (1-7) axis, which protects against angiotensin II-induced hypertension.

Confirming the prior observations in humans, Zhuofeng Lin and colleagues demonstrated that mice treated with angiotensin II had increased Fgf21 expression in the liver and raised serum levels of FGF21. Next, the researchers examined FGF21-knockout mice. Basal blood pressure and heart rate were similar in wild-type and knockout mice; however, angiotensin II-induced hypertension was found to be worse in the knockout mice compared with the wild-type mice, with significantly increased average systolic and diastolic pressures.

ACE2, which metabolizes angiotensin II to angiotensin (1-7), has a role in maintaining blood pressure homeostasis by negatively modulating the renin-angiotensin system. "In this project, we provide both in vivo and in vitro experimental data to clarify how ACE2 mediates the protective effect of FGF21 against angiotensin II-induced hypertension," explains Lin. "It is the first time the relationship between these two important proteins has been identified." In a series of experiments, Lin and colleagues showed that FGF21 induces the activity of ACE2 derived from adipocytes and renal cells, which protected against

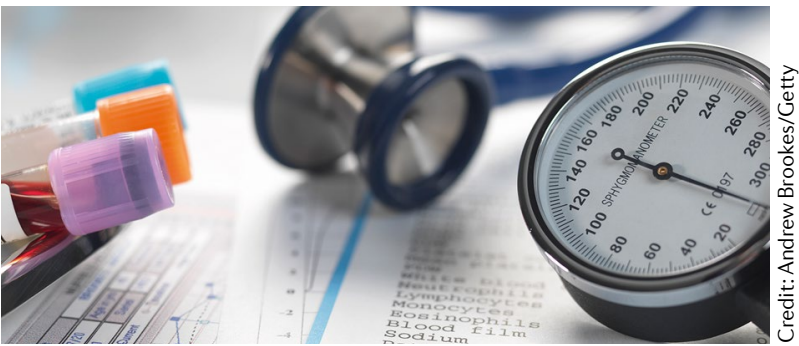

hypertension caused by angiotensin II, and also reversed vascular damage.

The authors note that their
ACE2

mediates the

protective

effect of

FGF21 against angiotensin

II-induced hypertension findings need to be confirmed in large non-human animals and in clinical studies. "The molecular mechanisms of FGF21 protecting against salt-induced hypertension need further investigation," adds Lin. The researchers are now planning to focus on these questions. "Our study may raise the possibility that FGF21 or its agonists are more effective for the treatment of hypertension than for diabetes," concludes Lin.

Claire Greenhill

ORIGINAL ARTICLE Pan, X. et al. FGF21 prevents angiotensin II-induced hypertension and vascular dysfunction by activation of ACE2/angiotensin-(1-7) axis in mice. Cell Metab. https://doi.org/10.1016/j. cmet.2018.04.002 (2018)

\section{CANCER METABOLISM}

\section{ALDOB promotes liver metastases development}

C6

Lack of

ALDOB

expression ...

suppressed

the

development

of liver

metastases
New research by Xiling Shen and colleagues shows that the hepatic microenvironment causes liver metastases of colorectal cancer in mice to upregulate fructose-bisphosphate aldolase $B$ (ALDOB), an enzyme involved in fructose metabolism, which subsequently fuels the growth of the hepatic metastases. In addition, dietary restriction of fructose has the same suppressive effect on the growth of liver metastases.

Metastatic cancer cells and the primary tumour have been thought to have similar functions. "Hence, we investigated whether metastatic cancer cells needed to adapt to their new microenvironment," explains Shen. To recapitulate the human disease, the researchers injected human colorectal cancer cells into the colons of immunodeficient mice, where they formed primary tumours that metastasized to the liver and lungs. Metabolic and transcriptomic analyses showed that ALDOB was upregulated in the liver metastases compared with the primary tumour. "Interestingly, the liver is a major metabolic site for fructose," explains Shen.

Next, the researchers overexpressed or knocked down ALDOB expression in liver metastatic cancer cells in vitro and showed that cancer cell development changed substantially when grown in cell culture medium containing fructose, but not in medium containing glucose. Trace analysis using isotope-labelled fructose revealed that metabolized fructose fuelled the growth of the liver metastases.

Shen and colleagues knocked down ALDOB expression in human colorectal cancer cells and injected the cells into mouse colons. Lack of ALDOB expression decreased the proliferation of cancer cells in the liver and suppressed the development of liver metastases. This inhibition was absent in control cells that expressed ALDOB.

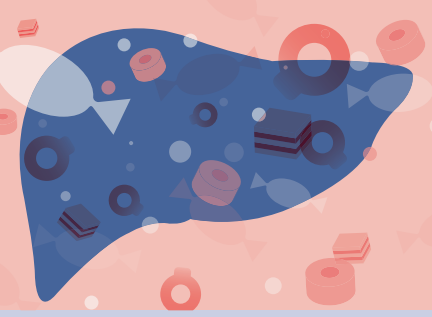

Credit: Jennie Vallis/Macmillan Publishers Limited

Finally, the researchers injected human colorectal cancer cells directly into the colons of mice and then fed them either a regulated high-fructose diet or a regulated diet without fructose. Mice fed the high-fructose diet had increased liver metastases compared with mice fed a diet of no fructose. These findings show that restricting dietary fructose also suppressed the development of liver metastases.

"Our next goal is to understand how fructose promotes tumour growth besides being a carbon source," concludes Shen.

Ivone Leong

ORIGINAL ARTICLE Bu, P. et al. Aldolase

B-mediated fructose metabolism drives metabolic reprogramming of colon cancer liver metastasis. Cell Metab. 27, 1-14 (2018) 